\section{Algoritmos de diagnóstico}

A TEP apresenta significativa mortalidade, sobretudo nas primeiras horas de sua apresentação. ${ }^{(1)} \mathrm{Na}$ verdade, o episódio que leva a sintomatologia inicial é um aviso de uma possível recorrência fatal. ${ }^{(2)}$ Portanto, exige-se rapidez no manejo dos pacientes.

0 espectro de apresentação de TEP é muito amplo. Ocorre desde um episódio assintomático em pacientes com TVP até a morte súbita, passando pela síndrome pleurítica, dispneia isolada e colapso circulatório. ${ }^{(3)}$ As manifestações irão depender da carga embólica pulmonar, do estado cardiopulmonar prévio do paciente e do grau de liberação de mediadores neuro-humorais. ${ }^{(4)}$ Por exemplo, uma TEP segmentar em paciente com DPOC grave pode levar à parada cardiocirculatória, enquanto uma obstrução de $30 \%$ da circulação pulmonar num paciente jovem, previamente hígido, em pós-operatório de cirurgia ortopédica, pode ser paucissintomática.

Diversas sociedades médicas têm elaborado algoritmos de manejo de pacientes com TEP, de complexidade diversa, apresentando diferenças, sobretudo nos pontos controversos na literatura. ${ }^{(5-7)}$ As recomendações finais dependem basicamente de como as evidências são valorizadas e da opinião dos especialistas do respectivo consenso.

Na elaboração dos algoritmos de diagnóstico, é preciso levar em conta os seguintes fatores: 1) gravidade do episódio de TEP (presença de instabilidade clínica e/ou hemodinâmica); 2) doença cardiopulmonar prévia significativa; 3) disponibilidade dos recursos diagnósticos; 4) disponibilidade de métodos terapêuticos; 5) o grau de invasividade dos procedimentos; 6) história prévia de TEP; 7) contraindicações ao tratamento farmacológico (heparinas e trombolíticos); 8) risco de transporte intra-hospitalar para a realização dos exames; 9) experiência da equipe médica no atendimento de pacientes com TEP; e 10) nível de evidência da recomendação na literatura. A história de TEP ou TVP prévias ao episódio atual poderá dificultar o diagnóstico da recorrência atual, sobretudo se não há exames de imagem após o tratamento do primeiro episódio mostrando reperfusão ou sequela permanente. ${ }^{(8)}$

No Quadro 9 estão sumarizadas as estratégias que, à luz da probabilidade clínica, permitem confirmar ou excluir TEP aguda com segurança. Outras combinações de resultados obrigam o prosseguimento da investigação. ${ }^{(9,10)}$
É conhecido que o maior fator prognóstico para a mortalidade na TEP é o grau de instabilidade hemodinâmica, representada por hipotensão, síncope (sinal de baixo débito) ou DVD significativa. ${ }^{(11)}$ Pacientes sem instabilidade hemodinâmica e sem DVD, quando tratados adequadamente, apresentam taxa de mortalidade relacionada à TEP quase nula. No outro polo, pacientes que apresentam colapso circulatório inicial ou deterioração clínica durante o episódio apresentam taxa de mortalidade que se aproxima a $32 \% .{ }^{(12)}$ Sendo assim, é essencial abordar esses grupos de pacientes de forma diferente.

\section{Pacientes clinicamente estáveis}

Considerando-se que TEP e TVP são polos da mesma doença, a saber, TEV, pacientes clinicamente estáveis com sintomas torácicos agudos e com sintomas e sinais de TVP deverão realizar inicialmente uma avaliação do sistema venoso profundo do membro afetado, já que a sensibilidade e a especificidade do método nesse contexto são elevadas (maior que 90\%). ${ }^{(13,14)}$ lsso permite o diagnóstico de TEV em caso de resultado positivo e o respectivo tratamento (anticoagulação, se não houver contraindicação). Entretanto, se o resultado foi negativo e houver suspeita de TEP, deve-se avaliar o comprometimento pulmonar, não sendo possível excluí-la somente por ecografia venosa normal.

Quando surge a suspeita criteriosa de TEP aguda, com base nos sintomas e sinais (como dispneia, taquipneia ou dor torácica) sem causa aparente do ponto de vista clínico ou nos exames de apoio iniciais (radiografia de tórax, ECG e gasometria arterial), é imprescindível o estabelecimento da probabilidade clínica. Atualmente, tendo em vista a variabilidade da experiência pessoal no atendimento de pacientes com TEP, recomenda-se a utilização de escores de predição clínica validados. ${ }^{(15)}$ Entre eles, o mais estudado e aplicável, tanto em pacientes que chegam à emergência quanto naqueles já hospitalizados, é o Escore Canadense (de Wells). Apesar de inicialmente dividido em três níveis, estudos mais recentes têm dicotomizado o escore (TEP improvável: escore $\leq 4$; ou TEP provável: escore > 4) na abordagem inicial, simplificando as decisões. ${ }^{(16)}$ Pacientes com escore $\leq 4$ deverão ser submetidos à dosagem de dímeros $\mathrm{D}$ de alta sensibilidade (ELISA, VIDAS, hemaglutinação ou Liatest). Sendo essa com níveis abaixo do valor normal, TEP pode ser excluída com segu- 
Quadro 9 - Estratégias definidoras de TEP aguda.

\begin{tabular}{|ll|}
\hline & Confirmação de TEP \\
\hline Probabilidade clínica alta & - Cintilografia pulmonar de alta probabilidade para TEP \\
& - Cintilografia pulmonar de probabilidade intermediária para TEP \\
& - Angio-TC positiva para TEP (segmentar ou maior) \\
& - Arteriografia convencional positiva \\
Probabilidade clínica intermediária & - Cintilografia pulmonar anormal, não de alta probabilidade e US com \\
& sinais de TVP \\
& - Angio-TC positiva para TEP (segmentar ou maior) \\
& - Arteriografia convencional positiva para TEP \\
Probabilidade clínica baixa & - Arteriografia convencional positiva para TEP \\
\hline & \multicolumn{1}{c|}{ Exclusão de TEP } \\
\hline Probabilidade clínica alta & - Arteriografia convencional negativa para TEP \\
Probabilidade clínica intermediária & - Dosagem de dímeros D normal (alta sensibilidade) \\
& - Cintilografia pulmonar normal \\
& - Cintilografia pulmonar de baixa probabilidade para TEP e US sem sinais \\
& de TVP \\
& - Angio-TC unidetector negativa para TEP e US sem sinais de TVP \\
& - Angio-TC multidetector negativa para TEP \\
& - Arteriografia convencional negativa para TEP \\
Probabilidade clínica baixa & Dosagem de dímeros D normal \\
& - Cintilografia pulmonar normal \\
& - Cintilografia pulmonar de baixa probabilidade para TEP \\
& - Angio-TC unidetector negativa para TEP \\
& - Angio-TC multidetector negativa para TEP \\
&
\end{tabular}

US: ultrassonografia. Adaptado de estudos anteriores. ${ }^{(10,11)}$

rança e sem a necessidade de realização de exames de imagem adicionais. Em pacientes com escore $>4$ (suspeita intermediária ou alta), não há a necessidade de se solicitar a dosagem de dímeros $\mathrm{D}$, pois um valor normal não exclui TEP, sendo necessário prosseguir a investigação. Cabe lembrar que a especificidade da dosagem de dímeros $\mathrm{D}$ com ponto de corte de $500 \mathrm{ng} / \mathrm{mL}$ não é adequada para o uso assistencial, tendo em vista o grande número de resultados falsopositivos em contextos nos quais TEP é comum, como em pós-operatórios e na presença de neoplasias malignas ou de doenças clínicas exacerbadas. ${ }^{(17)}$

Convém lembrar que pacientes com suspeita clínica intermediária ou alta deverão receber anticoagulantes, quando não houver contraindicações, para reduzir o risco de recorrência durante o período da investigação diagnóstica. $\mathrm{Na}$ presença de contraindicações aos anticoagulantes, pode-se optar por $\mathrm{FVCl}$ temporários até que se confirme ou não o diagnóstico, apesar do custo elevado desses dispositivos. ${ }^{(18)}$
Hoje em dia, a angio-TC do tórax com multidetectores (sobretudo, com 8 ou mais canais) tem se tornado o exame de eleição para TEP, já que é mais disponível nos hospitais que a cintilografia pulmonar, além de permitir realizar diagnósticos alternativos a TEP caso o resultado seja negativo. ${ }^{(19,20)}$ Entretanto, a cintilografia pulmonar perfusional pode ser o método inicial de eleição em pacientes com radiografia normal (o que promove o aumento da acurácia em relação aos pacientes com doença pulmonar prévia), alergia a contraste iodado e/ou insuficiência renal. ${ }^{(21)}$ Os resultados da angio-TC ou da cintilografia pulmonar que sejam concordantes com a probabilidade clínica (isto é, angio-TC positiva ou cintilografia de alta probabilidade com probabilidade clínica alta, ou angio-TC negativa ou cintilografia de baixa probabilidade com probabilidade clínica baixa) permitem, respectivamente, confirmar ou excluir TEP com segurança. Já com resultados discordantes (isto é, angio-TC negativa ou cintilografia de baixa 
probabilidade com probabilidade clínica alta, ou angio-TC positiva ou cintilografia de alta probabilidade com probabilidade clínica baixa), há a necessidade de investigação adicional. ${ }^{(22)}$

Em pacientes clinicamente estáveis, o exame recomendado na sequência é a ecografia de compressão e/ou Doppler de sistema venoso profundo de membros inferiores (incluir membros superiores na presença de cateter venoso central), mesmo em indivíduos assintomáticos para TVP, já que a presença de trombose nesse local permite o diagnóstico nosológico de TEV e o respectivo tratamento. ${ }^{(10)}$ Métodos alternativos ao ecografia para o estudo do sistema venoso profundo são a venorressonância, a veno-TC e a flebografia convencional. ${ }^{(22)}$

Nos pacientes com estudo do sistema venoso profundo negativo que mantiverem a suspeita clínica de TEP, deverá ser realizada a arteriografia pulmonar, que, apesar de ser um método invasivo e com necessidade de uso de contraste, é seguro em centros experientes, desde que tomadas as devidas precauções (prevenção de nefropatia de contraste, estabilização antes do exame, uso de contraste não iônico e uso de arteriografia por subtração digital). ${ }^{(22)}$ Sendo a arteriografia pulmonar convencional o padrão ouro, o resultado positivo (isto é, defeitos de enchimento vascular) permite confirmar o diagnóstico de TEP, bem como o resultado negativo o afasta com segurança, podendo-se, nesse último caso, suspender a anticoagulação se essa havia sido iniciada.

É importante ressaltar que, para pacientes cujo diagnóstico de TEV foi excluído, se inicie a profilaxia primária (se isso ainda não tenha sido feito) e se mantenha a vigilância para a possibilidade de TVP ou TEP, pois, em geral, os pacientes estão em cenário de risco, tanto que a suspeita foi levantada.

\section{Pacientes clinicamente instáveis}

No caso de pacientes que estão clinicamente instáveis (hipotensão, síncope e/ou hipoxemia significativas) e quando há a suspeita de TEP (geralmente intermediária ou alta), há a necessidade de rapidez no diagnóstico para que o tratamento seja iniciado o mais breve possível, em especial quando o VD está demonstrando sinais diretos ou indiretos de falência, o que pode culminar em choque cardiogênico refratário e óbito. ${ }^{(4)}$ Atualmente, estão sendo testados biomarcadores, como a troponina e brain natriuretic peptide(BNP), para auxiliar na estratificação do risco de desfecho desfavorável na TEP. ${ }^{(23)}$

Nos pacientes com hipotensão, há o benefício do tratamento com trombolíticos. ${ }^{(24)}$ Caso haja contraindicação para o seu uso, as alternativas são a embolectomia por cateterismo ou mesmo a cirurgia aberta, que, em geral, tem alta mortalidade devido ao estado do paciente que é levado ao bloco cirúrgico. Sendo assim, é essencial uma rápida definição da presença ou não de TEP. Por isso, o método de eleição é a arteriografia convencional, já que em centros com experiência, durante a arteriografia, é possível realizar a embolectomia por cateter, unindo procedimentos diagnósticos e terapêuticos, muitas vezes evitando a embolectomia cirúrgica. ${ }^{(21,25)}$

Nos pacientes que são candidatos a trombólise, pode-se optar inicialmente por um método diagnóstico não invasivo. No caso de pacientes com risco elevado de transporte intra-hospitalar (em uso de vasopressor em altas doses, em ventilação mecânica com alta $\mathrm{FiO}_{2}$ ou com parada cardiorrespiratória na apresentação inicial de TEP) pode-se usar métodos à beira do leito, sobretudo o ecocardiograma, idealmente, o transesofágico. ${ }^{(26,27)}$ Esse método, além da boa sensibilidade para trombos centrais, permite o diagnóstico alternativo de condições que simulam a instabilidade hemodinâmica da TEP, como a dissecção aórtica, o infarto do miocárdio com complicação mecânica e o tamponamento pericárdico, situações nas quais o uso de trombolíticos poderia ser desastroso. 0 ecodopplercardiograma de sistema venoso profundo de membros inferiores (incluir membros superiores na presença de sinais clínicos de TVP ou quando do uso de cateter venoso central), num contexto de paciente com suspeita de TEP e instabilidade clínica, permite o diagnóstico operacional de TEV. Caso esses métodos à beira do leito não demonstrarem TEP e a suspeita seja mantida, ou no caso de pacientes que podem ser transportados ao departamento de radiologia, o método de escolha é a angio-TC de tórax. Para os casos duvidosos após essa etapa, deverá ser realizado o teste padrão ouro, a arteriografia pulmonar. ${ }^{(6,28)}$

\section{Recomendações para a investigação de TEP}

Os algoritmos apresentados a seguir são uma sugestão de conduta, deverão ser adaptados às diferentes instituições de saúde de acordo com a respectiva disponibilidade e não substituem o 
julgamento do médico assistente, o qual deverá utilizar os conceitos apresentados à luz das particularidades do paciente para a decisão da conduta final. Dessa forma, é essencial que cada hospital faça seus protocolos de atendimento locais, embasados nas recomendações aqui apresentadas.

Os pontos essenciais desses algoritmos são os seguintes: 1) valorização da probabilidade clínica como primeiro método diagnóstico (que deverá ser estimada, se possível, por escores validados) antes da solicitação dos exames confirmatórios; 2) estratificação dos pacientes em clinicamente estáveis e instáveis; 3) definição operacional de presença ou ausência de TEP por uma combinação da probabilidade clínica e dos resultados de exames não invasivos e invasivos; e 4) atrelamento entre os métodos diagnósticos e os recursos terapêuticos (Figuras 1 e 2).

Os seguintes pontos devem ser enfatizados:

1) As instituições de saúde devem elaborar protocolos de atendimento de pacientes com TEP aguda de acordo com a diretriz adaptada à realidade e à disponibilidade local.(D)

2) 0 algoritmo de abordagem dos pacientes com TEP aguda deve diferenciar pacientes que se apresentam clinicamente estáveis daqueles clinicamente instáveis (choque, hipotensão relativa, síncope ou hipoxemia). (B)

3) As decisões do manejo individual do paciente devem levar em conta, sobretudo, a instabilidade clínica, a disponibilidade local e as particularidades do caso.(D)

4) No caso de pacientes com suspeita de TEP, deve ser estabelecida a probabilidade pré-teste da doença, através do Escore Canadense de Wells para TEP. Caso o paciente apresente também sinais de TVP, recomenda-se o uso do Escore Canadense de Wells para TVP.(A)

5) Pacientes com suspeita clínica moderada ou alta devem receber heparinização plena enquanto realizam os exames diagnósticos.(B)

6) No caso de pacientes clinicamente estáveis com suspeita de TEP e que apresentam sinais clínicos de TVP (edema, calor e dor unilateral em extremidade), a investigação deve ser iniciada com ecografia de compressão e/ou Doppler de sistema venoso do membro acometido. A confirmação de TVP permite o tratamento de TEV. Caso o resultado seja negativo para TVP, deve-se proceder a investigação.(B)
7) Em pacientes com baixa suspeita clínica (Escore de Wells $\leq 4$ ) deverá ser solicitada a dosagem de dímeros $D$ (preferencialmente através dos métodos ELISA, látex automatizado ou hemaglutinação). Caso 0 resultado seja negativo (dosagem $<500 \mathrm{ng} / \mathrm{mL}$ ), o diagnóstico de TEP pode ser excluído sem a necessidade de investigação adicional. Para pacientes com níveis elevados de dímeros $\mathrm{D}$, deve-se continuar a investigação.(A)

8) A angio-TC de tórax com multidetectores (sobretudo $>8$ detectores) é o método preferencial de diagnóstico e permite observar diagnósticos alternativos na ausência de TEP.(A)

9) A cintilografia pulmonar perfusional, combinada com a radiografia de tórax ou a cintilografia inalatória, é o método preferencial em pacientes com insuficiência renal prévia, história de alergia ao contraste e gestantes, sobretudo se não houver doença cardiopulmonar prévia e a radiografia de tórax for normal.(B)

10) Resultados da angio-TC de tórax ou da cintilografia pulmonar concordantes (isto é, angio-TC positiva ou cintilografia de alta probabilidade com probabilidade clínica alta, ou angio-TC negativa ou cintilografia de baixa probabilidade com probabilidade clínica baixa), permitem, respectivamente, confirmar ou excluir o diagnóstico de TEP com segurança. Resultados discordantes (isto é, angio-TC negativa ou cintilografia de baixa probabilidade com probabilidade clínica alta, ou angio-TC positiva ou cintilografia de alta probabilidade com probabilidade clínica baixa), há a necessidade de investigação adicional.(A)

11) Pacientes clinicamente estáveis com resultados da angio-TC ou cintilografia discordantes da probabilidade clínica, deverão ser submetidos à ecodopplercardiograma do sistema venoso profundo. Caso o resultado comprove TVP, está confirmado o diagnóstico de TEV. Na presença de resultado negativo, recomenda-se a realização de arteriografia pulmonar.(A)

12) A arteriografia pulmonar é o padrão ouro de diagnóstico. 0 resultado positivo confirma diagnóstico de TEP, e resultado negativo afasta TEP com segurança.(A)

13) Pacientes clinicamente instáveis com risco para transporte intra-hospitalar deverão ser submetidos a ecocardiograma transesofágico à beira do leito. 0 diagnóstico de TEP por sinais diretos ou a presença de 


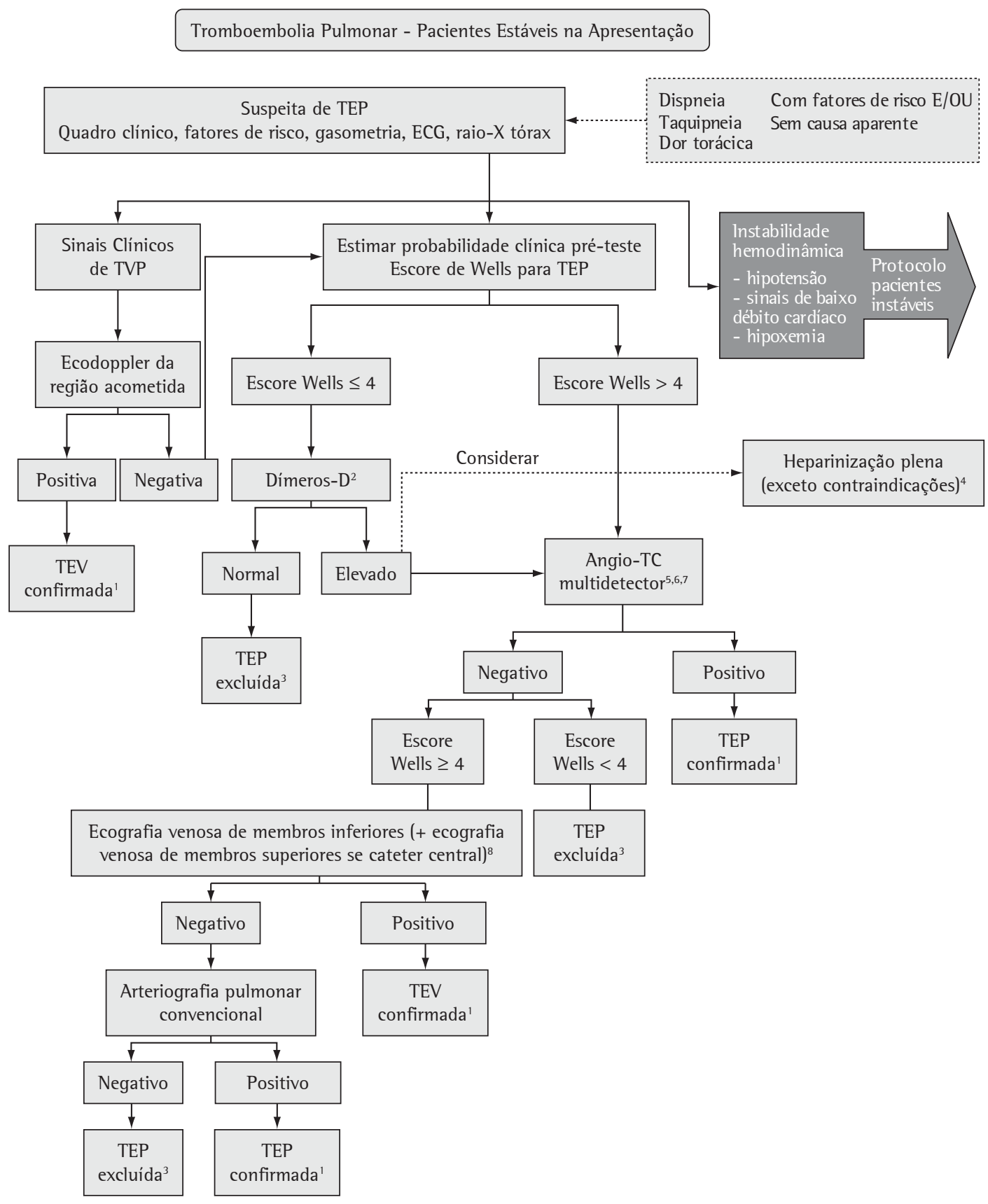

'Com TEP-TEV confirmada, complementar a estratificação de risco (Ver algoritmo 3)

${ }^{2}$ Dosagem de dímeros D por método com alta sensibilidade (> 90\%): ELISA, VIDAS

${ }^{3}$ Procurar outro diagnóstico + profilaxia primária para TEV + manter vigilância para TEV

${ }^{4}$ Nos casos com contraindicação a heparina, considerar colocação de filtro de veia cava inferior, se possível tipo temporário, até a confirmação da TEP (no contexto de alta suspeita clínica)

${ }^{5} \mathrm{Se}$ a angio-TC for negativa e a suspeita clínica for alta, pode-se combinar veno-TC no mesmo exame

${ }^{6}$ Se alergia ao contraste, insuficiência renal ou gestação, o exame inicial indicado é a cintilografia pulmonar perfusional (comparada com radiografia de tórax)

${ }^{7}$ Angio-TC helicoidal com um detector com boa qualidade técnica (boa opacificação da circulação pulmonar) pode ser suficiente para a maioria das situações

${ }^{8}$ Outros métodos para diagnóstico de TVP: venorressonância, veno-TC ou flebografia convencional

Figura 1 - Algoritmo de diagnóstico de TEP em pacientes clinicamente estáveis. 


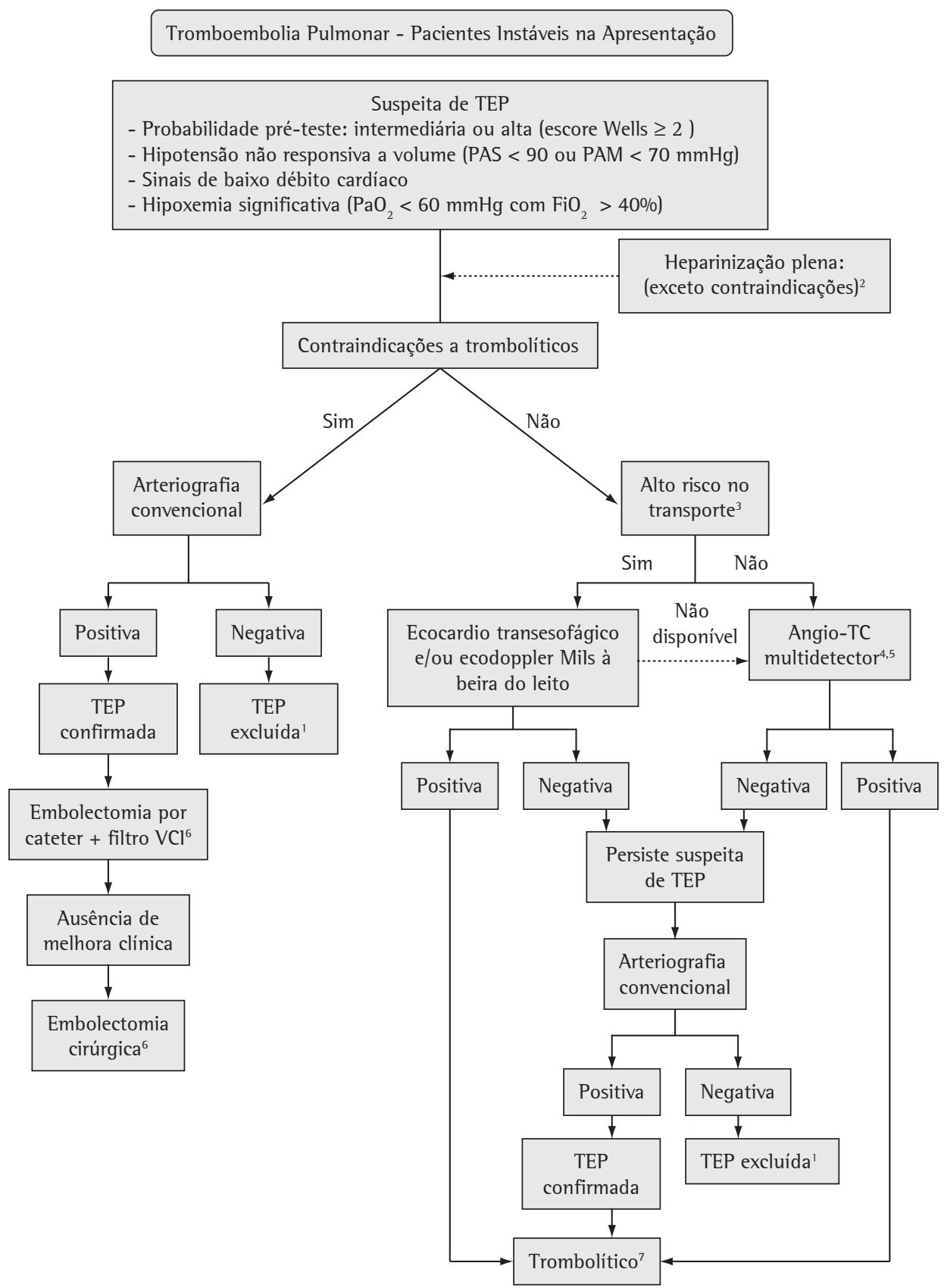

\footnotetext{
' Procurar outro diagnóstico + profilaxia primária para TEV + manter vigilância para TEV

${ }^{2}$ Se for utilizar trombolítico imediatamente, não utilizar heparina concomitante. Caso contraindicação a heparina, considerar colocação de filtro $\mathrm{VCl}$, se possível do tipo temporário, até a confirmação da TEP

${ }^{3}$ Uso de vasopressor em moderadas ou altas doses, VM com FiO2 > 0.6, PCR na apresentação

${ }^{4}$ Considerar ecocardiograma transesofagico se alergia ao contraste iodado ou insuficiencia renal grave

${ }^{5}$ Angio-TC helicoidal com um detector mostrando imagens com boa qualidade técnica (boa opacificação da circulação pulmonar) pode ser suficiente para a maioria das situações

${ }^{6}$ Depende da disponibilidade local. Se necessário e paciente com condições de transporte, considerar transferência para centro de referência

${ }^{7}$ Iniciar HNF i.v. em infusão contínua (sem bolus), conforme TTPa seriado após uso do trombolítico
}

Figura 2 - Algoritmo de diagnóstico de TEP em pacientes clinicamente instáveis. PAS: pressão arterial sistólica; PAM: pressão arterial média; Ecocardio: ecocardiograma; Mils: membros inferiores; $\mathrm{VCl}$ : veia cava inferior. 
sinais indiretos na ausência dos principais diagnósticos diferenciais (tamponamento cardíaco, dissecção de aorta, IAM com complicação mecânica) permitem o diagnóstico assistencial para fins de tratamento de TEP.(C)

14) Pacientes com risco de transporte, mas que não puderem ser submetidos ao exame à beira do leito, ou se esse tenha um resultado inconclusivo, devem ser submetidos à arteriografia pulmonar, sobretudo se na instituição há um médico que realiza embolectomia por cateter. Na impossibilidade de arteriografia convencional, a angio-TC com multidetectores é um método aceitável.(C)

\section{Referências}

1. Alpert JS, Dalen JE. Epidemiology and natural history of venous thromboembolism. Prog Cardiovasc Dis. 1994;36(6):417-22.

2. Calder KK, Herbert M, Henderson SO. The mortality of untreated pulmonary embolism in emergency department patients. Ann Emerg Med. 2005;45(3):302-10.

3. Stein PD, Henry JW. Clinical characteristics of patients with acute pulmonary embolism stratified according to their presenting syndromes. Chest. 1997;112(4):974-9.

4. Wood KE. Major pulmonary embolism: review of a pathophysiologic approach to the golden hour of hemodynamically significant pulmonary embolism. Chest. 2002;121(3):877-905.

5. Hirsh J, Hoak J. Management of deep vein thrombosis and pulmonary embolism. A statement for healthcare professionals. Council on Thrombosis (in consultation with the Council on Cardiovascular Radiology), American Heart Association. Circulation. 1996;93(12):2212-45.

6. Tapson VF, Carroll BA, Davidson BL, Elliott CG, Fedullo $\mathrm{PF}$, Hales CA, et al. The diagnostic approach to acute venous thromboembolism. Clinical practice guideline. American Thoracic Society. Am J Respir Crit Care Med. 1999;160(3):1043-66.

7. British Thoracic Society Standards of Care Committee Pulmonary Embolism Guideline Development Group. British Thoracic Society guidelines for the management of suspected acute pulmonary embolism. Thorax. 2003;58(6):470-83.

8. Menna Barreto SS, Gazzana MB. Tromboembolia pulmonar. In: Menna Barreto SS, Vieira SR, Pinheiro CT. Rotinas em terapia intensiva. Porto Alegre: Artmed; 2000. p. 193-215.

9. Fedullo PF, Tapson VF. Clinical practice. The evaluation of suspected pulmonary embolism. N Engl J Med. 2003;349(13):1247-56.

10. Roy PM, Colombet I, Durieux P, Chatellier G, Sors H, Meyer G. Systematic review and meta-analysis of strategies for the diagnosis of suspected pulmonary embolism. BMJ. 2005;331(7511):259.

11. Torbicki A, Perrier A, Konstantinides S, Agnelli G, Galiè $\mathrm{N}$, Pruszczyk P, et al. Guidelines on the diagnosis and management of acute pulmonary embolism: the Task Force for the Diagnosis and Management of Acute Pulmonary Embolism of the European Society of Cardiology (ESC). Eur Heart J. 2008;29(18):2276-315.
12. Goldhaber SZ, Visani L, De Rosa M. Acute pulmonary embolism: clinical outcomes in the International Cooperative Pulmonary Embolism Registry (ICOPER). Lancet. 1999;353(9162):1386-9.

13. Kreit JW. The impact of right ventricular dysfunction on the prognosis and therapy of normotensive patients with pulmonary embolism. Chest. 2004;125(4):1539-45.

14. Zierler BK. Ultrasonography and diagnosis of venous thromboembolism. Circulation. 2004;109(12 Suppl 1):19-14.

15. Chunilal SD, Eikelboom JW, Attia J, Miniati M, Panju AA, Simel DL, et al. Does this patient have pulmonary embolism? JAMA. 2003;290(21):2849-58.

16. van Belle A, Büller HR, Huisman MV, Huisman PM, Kaasjager K, Kamphuisen PW, et al. Effectiveness of managing suspected pulmonary embolism using an algorithm combining clinical probability, D-dimer testing, and computed tomography. JAMA. 2006;295(2):172-9.

17. Caprini JA, Glase CJ, Anderson CB, Hathaway K. Laboratory markers in the diagnosis of venous thromboembolism. Circulation. 2004;109(12 Suppl 1):14-8.

18. Büller HR, Agnelli G, Hull RD, Hyers TM, Prins MH, Raskob GE. Antithrombotic therapy for venous thromboembolic disease: the Seventh ACCP Conference on Antithrombotic and Thrombolytic Therapy. Chest. 2004;126(3 Suppl):401S-428S. Erratum in: Chest. 2005 Jan;127(1):416.

19. Quiroz R, Kucher N, Zou KH, Kipfmueller F, Costello P, Goldhaber SZ, et al. Clinical validity of a negative computed tomography scan in patients with suspected pulmonary embolism: a systematic review. JAMA. 2005;293(16):2012-7.

20. Filipek MS, Gosselin MV. Multidetector pulmonary CT angiography: advances in the evaluation of pulmonary arterial diseases. Semin Ultrasound CT MR. 2004;25(2):83-98.

21. Dalen JE. Pulmonary embolism: what have we learned since Virchow? Natural history, pathophysiology, and diagnosis. Chest. 2002;122(4):1440-56.

22. Andrews RT. Contrast peripheral phlebography and pulmonary angiography for diagnosis of thromboembolism. Circulation. 2004;109(12 Suppl 1):122-7.

23. Kucher N, Goldhaber SZ. Cardiac biomarkers for risk stratification of patients with acute pulmonary embolism. Circulation. 2003;108(18):2191-4.

24. Wan S, Quinlan DJ, Agnelli G, Eikelboom JW. Thrombolysis compared with heparin for the initial treatment of pulmonary embolism: a meta-analysis of the randomized controlled trials. Circulation. 2004;110(6):744-9.

25. Rahimtoola A, Bergin JD. Acute pulmonary embolism: an update on diagnosis and management. Curr Probl Cardiol. 2005;30(2):61-114.

26. Warren J, Fromm RE Jr, Orr RA, Rotello LC, Horst HM; American College of Critical Care Medicine. Guidelines for the inter- and intrahospital transport of critically ill patients. Crit Care Med. 2004;32(1):256-62.

27. Goldhaber SZ. Echocardiography in the management of pulmonary embolism. Ann Intern Med. 2002;136(9):691-700.

28. Institute for Clinical Systems Improvement. Venous Thromboembolism Diagnosis and Treatment. Bloomington: Institute for Clinical Systems Improvement; 2009. 